\title{
Synthesis and Characterization of Surfactant Stabilized Trimetallic Au-Ag-Pd Nanoparticles for Heck Coupling Reaction
}

\author{
P. Venkatesan*, J. Santhanalakshmi \\ Department of Physical Chemistry, University of Madras, Guindy Campus, A.C.Tech., Chennai, 600025, Tamil Nadu, India
}

\begin{abstract}
Colloidal dispersions of trimetallic nanoparticles (tnp) are synthesized by chemical method and compositionally four different tnp are prepared and particle sizes are characterized by UV-vis, HRTEM and XPS measurements. The XPS studies provide proof of the mode of binding that occurs in the nanoparticle surface. The catalytic activities of tnp are tested by Heck C-C coupling reaction. The product yields and recyclability of the recovered catalysts are studied. Tnp exhibited better catalysis than mono and bimetallic nanoparticles, which may be due to electronic effects of the Au-Ag core on to the Pd shell atoms.
\end{abstract}

Keywords Trimetallic Nanoparticles; XPS Study; Core-Shell Nanoparticles; C-C Coupling Reaction; Gas Chromatography; Catalysts

\section{Introduction}

Colloidal dispersions of bi and multi metallic nanoparticles have garnered a lot of attention in recent years, particularly in the areas of optoelectronic applications and catalysis[1-5]. In the realm of optoelectronic applications, metal nanoparticles have interesting size and shape- dependent optical and electronic properties that can be suitably modulated by the addition of another metal[6]. For catalytic applications, the expectation is that they will have better catalytic properties than the component metals alone or even have new properties[7]. Chemical preparation of these colloidal multi metallic systems usually involves simultaneous and sequential reduction of the precursor ions and latter usually gives rise to core-shell structures. In both cases polymers, surfactants, ligands are added during the reduction process to prevent aggregation of the formed colloidal nanoparticles[8-10].

Chen-Sheng Yeh et al. were among the first to use laser irradiation to synthesize trimetallic nanoparticles[11]. They used a Nd:YAG pulsed laser operated at a $10 \mathrm{~Hz}$ to synthesize tnp from aqueous solutions containing gold, silver and palladium. In our group, Santhanalakshmi et al. have reported that to prepare mono metallic $\mathrm{Au}, \mathrm{Ag}, \mathrm{Pd}$, and bimetallic $\mathrm{Au}-\mathrm{Ag}, \mathrm{Ag}-\mathrm{Pd}$, and $\mathrm{Au}-\mathrm{Pd}$ core-shell nanoparticles

* Corresponding author:

venkatesanorg@gmail.com (P. Venkatesan)

Published online at http://journal.sapub.org/pc

Copyright (C) 2012 Scientific \& Academic Publishing. All Rights Reserved by chemical reduction of metal ions in aqueous solution $[12,13]$, particle sizes and distribution are governed by the reduction rate, with faster rates leading to smaller and more uniform particles. Haverkamp et al. have synthesized tnp of gold, silver and copper with a narrow size distribution by irradiation of an aqueous solution containing both $\mathrm{Au}(\mathrm{III})$, $\mathrm{Ag}(\mathrm{I})$ and $\mathrm{Cu}(\mathrm{II})$ ions. They reported alloy like core-shell morphology for the resulting nanoparticles with a $\mathrm{Cu}$ shell over the $\mathrm{Au}, \mathrm{Ag}$ core tnp with $\mathrm{Au}$ core, $\mathrm{Ag}$ and $\mathrm{Cu}$ shell have also been synthesized by Haverkamp et al. using successive irradiation of the precursors copper chloride, silver nitrate and gold chloride in plants materials[14]. Xin Zhag et al. have also reported the synthesis of tnp of Pt-Ru-Mo in the presence of microemulsions[15], Au-Pt-Rh in the presence of poly(N-vinyl-2-pyrrolidone)[16] and Zhang et al have also prepared $\mathrm{Pt}-\mathrm{Ru}-\mathrm{Co}$ trimetallic clusters by the reduction of an aqueous $\mathrm{Pt}-\mathrm{Ru}-\mathrm{Co}$ precursor solution with a parallel micro-emulsion system[17].

In this paper, the synthesis of Au-Ag-Pd trimetallic nanoparticles in the chemical method by the simultaneous reduction of $\mathrm{HAuCl}_{4}, \mathrm{AgNO}_{3}$ and $\mathrm{H}_{2} \mathrm{PdCl}_{4}$ with reducing agent was studied using CTAB as the capping agent. The size, structure, optical properties and composition distribution of the resultant nanoparticles were characterized by UV-vis, TEM and XPS. The catalytic efficiency of the environmentally benign Pdnp and that of the four compositionally different tnp are monitored by Heck coupling reaction shown in Scheme 1. The products yield, reaction time and recyclability of the catalyst are the parameters derived from the reaction conducted separately using the Pdnp and four tnp catalysts. 


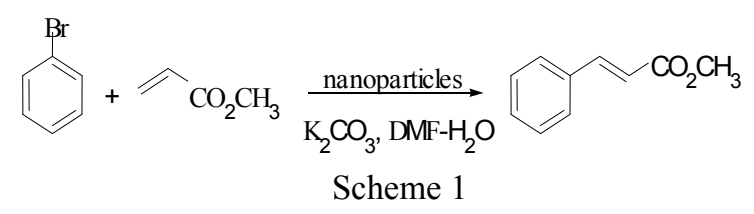

\section{Experimental}

\subsection{Preparation of Au-Ag-Pd trimetallic nanoparticles}

Our laboratory recently developed a methodology for sequential reduction and preparing core-shell nanoparticles by wet chemical method of mixtures consisting three metal colloids, e.g. $\mathrm{Au}-\mathrm{Ag}-\mathrm{Pd}[18]$. In view of our successful preparation of tnp, we took the next step and conducted the first trial of simultaneous reduction of three metals in chemical methods in $\mathrm{Au}-\mathrm{Ag}-\mathrm{Pd}$ colloidal mixtures.

The colloidal dispersions of surfactant protected tnp were prepared by refluxing of the aqueous solution of $\mathrm{HAuCl}_{4}$, $\mathrm{AgNO}_{3}$ and $\mathrm{PdCl}_{2}$ in the presence of CTAB. The colloidal dispersion of 1:1:1 precursor metal salts are mixed at refluxed temperature in the designated ratio at one hour and the molar ratio of monomer unit of CTAB against total metal (R) were kept 30 in the present experiments. The mixed dispersions are kept stirring at least for a $24 \mathrm{hrs}$ at room temperature to complete the self-organization reaction. The tnp with different compositions molar ratio $(1: 1: 0.5,1: 0.5: 1$ and $0.5: 1: 1)$ of the precursor metal salts of gold, silver and palladium could be prepared without any precipitation above the similar method. The solutions of all the compositions are very stable over extended periods of time.

\subsection{Heck coupling reaction - Catalytic study}

Methyl acrylate $(1.05 \mathrm{mmol})$, bromobenzene $(1.0 \mathrm{mmol})$, $\mathrm{K}_{2} \mathrm{CO}_{3}(3.0 \mathrm{mmol})$ and $15 \mathrm{~mL}$ of $3: 1 \mathrm{v} / \mathrm{v}$ DMF-water solvent mixture are added into a three necked round bottomed flask with continuous stirring under $\mathrm{N}_{2}$ atmosphere. 0.5 mol \% nanoparticle catalysts are added and temperature is maintained at $120^{\circ} \mathrm{C}$. The progress of the reaction was monitored with gas chromatography. After completion of the reaction, $0.1 \mathrm{M} \mathrm{HCl}$ solution and 4-fold excess of $\mathrm{CH}_{2} \mathrm{Cl}_{2}$ are added so that, the nanoparticles catalyst and the products are correspondingly separated into the $\mathrm{pH}$ responsive aqueous and organic phases respectively. The catalyst was collected by ultracentrifugation and reused for the next cycle of coupling reaction.

\section{Results and Discussion}

The completion of formation reactions of mono metallic gold, bimetallic Au-Ag and trimetalli Au-Ag-Pd (1:1:1 mole ratio) nanoparticles were followed by UV-vis spectra as a function of time (Fig.1). The absorption spectra of the mixtures changed as the duration time was increased, the surface plasmon resonances peak (spr) bands moved closer to one another and merged into a single peak over the course of the time intervals. The mono metallic gold nanoparticles as conformed within $7 \mathrm{~min}$ and the bimetallic Au-Ag nanoparticles as conformed after $50 \mathrm{~min}$, further increased the time duration resulted in a continuous decrease in the absorption band until, eventually, no absorption peak was apparent after $60 \mathrm{~min}$ exposure. As the duration of the time was increased upto $24 \mathrm{hrs}$, there is no distinct change occurred in the shape of the absorbance.

Finally the color of the colloidal nanoparticles is dark brown as conformed formation of palladium coated trimetallic nanoparticles. The Pd nanoparticle shows a sharp absorbance peak cannot be observed over the entire range due to the brown colour of the solution. Successive reduction strategies are an effective method to prepare core-shell structure of bi and multi metallic nanoparticles. One of the metal salts is reduced first to form the core, and then a second metal is deposited on the surface of pre-formed monometallic nanoparticles to form the shell.

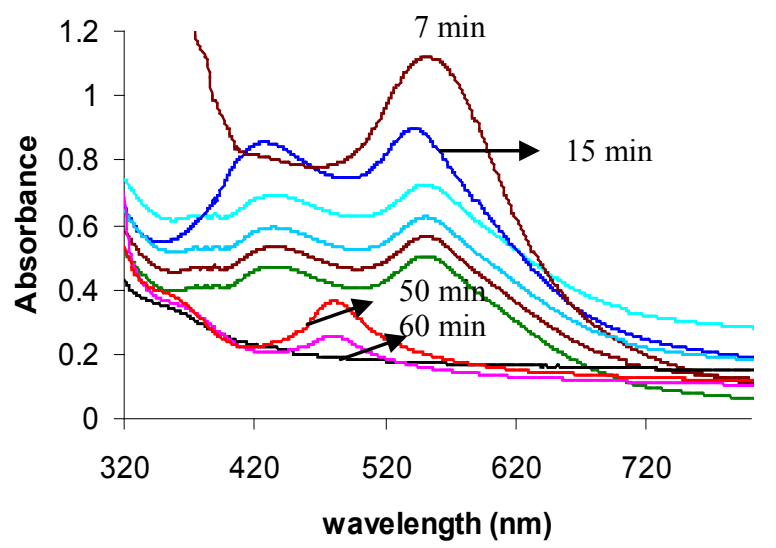

Figure 1. UV-Vis absorption spectra of CTAB stabilized trimetallic $\mathrm{Au}-\mathrm{Ag}-\mathrm{Pd}$ colloidal nanoparticles at different time intervals in aqueous medium at $25^{\circ} \mathrm{C}$

In Fig. 2 typical UV-vis spectra of various mole ration of tnp (a) $1: 1: 0.5$, (b) $1: 0.5: 1$, (c) $0.5: 1: 1$ and (d) $(1: 1: 1)$ in aqueous medium are given. The spr peaks are shoulder peak correspond to various tnp. No surface plasmon peak (spr) was observed for trimetallic nanoparticles.

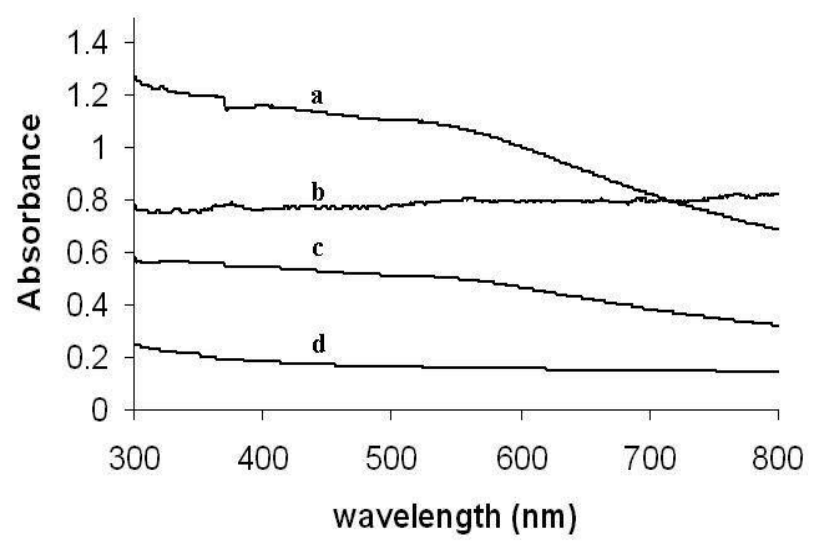

Figure 2. UV-Vis absorption spectra of $\mathrm{CTAB}$ stabilized trimetallic nanoparticles of various concentrations, (a) 1:1:0.5, (b) 1:0.5:1, (c) 0.5:1:1, and (d) $1: 1: 1$ respectively in aqueous medium at $25^{\circ} \mathrm{C}$ 
HRTEM photographs of aliquots of nanoparticle solution as in the preparation conditions drawn out each from the three different temperature regions viz: $25-40,50$ and $80^{\circ} \mathrm{C}$ is presented in Fig. 3a, 3b and 3c respectively. The particle sizes seem to increase with increase in temperature and also core-shell particles are found in the 50 and $80^{\circ} \mathrm{C}$ HRTEM photographs. The low temperature region $\left(<50^{\circ} \mathrm{C}\right)$ nanoparticles correspond to pure Aunp formations (initially). Hence Aunp, Au-Agnp, Au-Ag-Pd nanoparticle diameters correspond to $6.5,9.0$ and $13 \pm 0.5 \mathrm{~nm}$ respectively. Figure 3(e) shows the electron diffraction pattern obtained from a selected area of Fig.3(d). The bright rings with occasional bright spots are due to the presence of polycrystals. Regarding the variation of particle sizes of tnp with different compositions, there is no appreciable change in the size and the size growth is only within $10 \%$ of the 1:1:1 tnp.

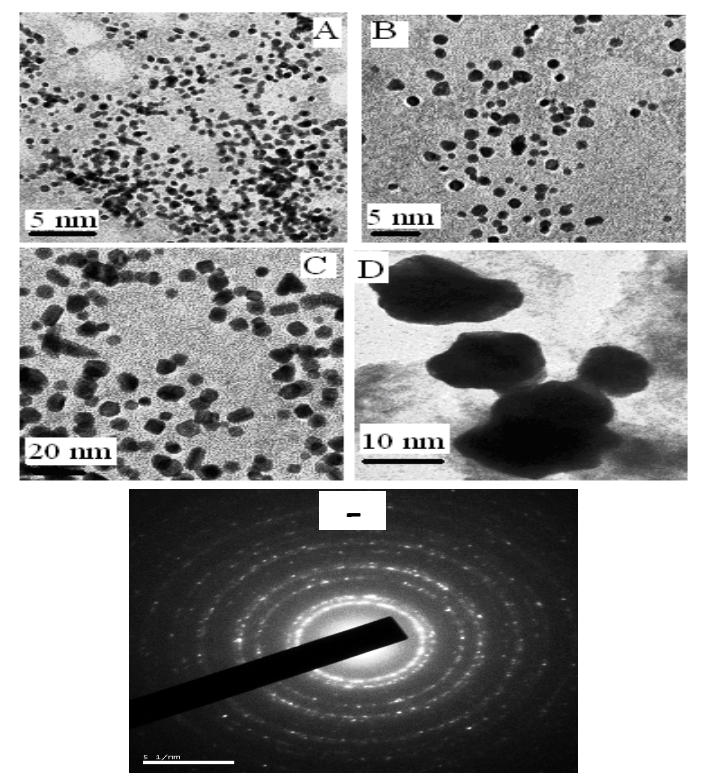

Figure 3. HRTEM images of aliquots in the tnp formation maintained at various temperatures (a) tnp at $35^{\circ} \mathrm{C} \mathrm{(b)} 50^{\circ} \mathrm{C}$, (c) $80^{\circ} \mathrm{C}$ and d) $80^{\circ} \mathrm{C}$ (magnified). Particle sizes: (a) 6.5 (b) 9.0 (c) $13.0 \pm 0.5 \mathrm{~nm}$ and (e) Electron diffraction pattern spectra of tnp $(1: 1: 1)$ in aqueous medium at $25^{\circ} \mathrm{C}$

To further investigate the oxidation states of the elements and the compositions in the outer part of trimetallic nanoparticles were measured. The XPS spectra of the Au4f, Ag3d and Pd3d regions of the CTAB-capped nanoparticles are shown in Fig.4. A doublet at (B.E) $335.4 \mathrm{eV}$ corresponding to $\mathrm{Au}-4 \mathrm{~d}$, in the respective multimetallic system represents metallic state of the colloids (Fig.4b). In Fig.4e, a doublet of $\mathrm{Pd}-3 \mathrm{~d}$ at $337.2 \mathrm{eV}$ with two narrow peaks is further evidence that palladium is present in its metallic state. No other oxidation states of palladium were detected in the sample. The latter decreased with the increase of Au-Ag-Pd ratio and should result from the formation of Pd-CTAB. Pd-CTAB should also be formed on the surface of Pd and Au-Ag-Pd particles (Fig.4c,d).

According to the intensities of XPS peaks, the elemental ratios of Au-Ag-Pd in the outer part of trimetallic nanoparticles could be obtained. It was obvious that the Pd atoms were enriched in the outer part of $\mathrm{Au}-\mathrm{Ag}-\mathrm{Pd}$ trimetallic nanoparticles, suggesting a structure of incomplete Au-Ag-core-Pd-shell. This was consistent with the observation of UV-vis absorption spectra. The control of composition distribution by feeding composition should be interesting and important for the preparation of catalysts.

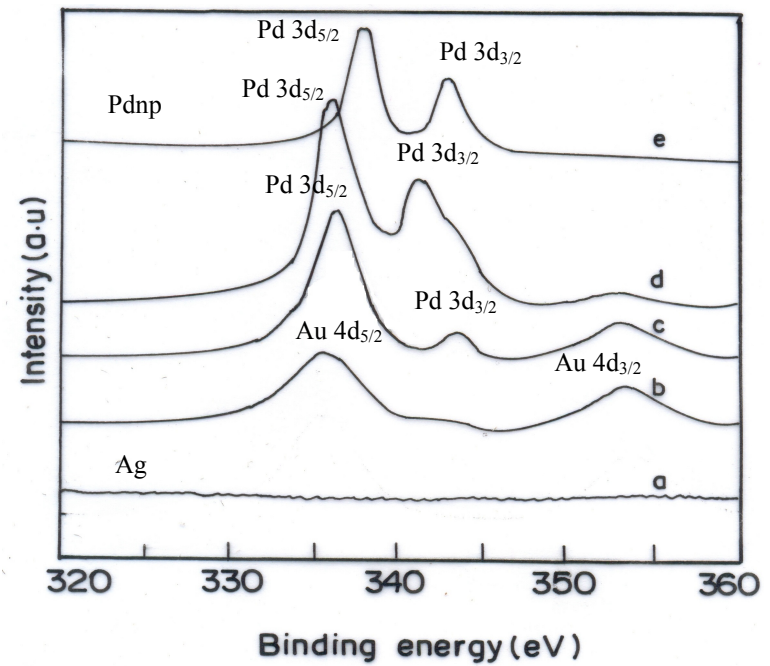

Figure 4. X-ray photoelectron spectra of individual mono metallic palladium and trimetallic colloidal nanoparticles at different mole ratio of (a) Au-Ag-Pd, 1:1:1, (b) Au-Ag-Pd, 1:1:1, (c) Au-Ag-Pd, 1:1:0.5, (d) Au-Ag-Pd, 1:1:1 and (e) Pdnp respectively at $25^{\circ} \mathrm{C}$

\section{Catalytic-activity tests: Heck reaction}

Recent reports showed that the trimetallic colloids are constituted of three distinct metals. Such compounds often showed better activity and selectivity compared to mono and bimetallic colloids [19]. In the present work, the product yields were determined by gas chromatography. Each catalytic reaction was carried out in at least two runs. Both plots reveal that using $0.5 \mathrm{~mol} \%$ tnp (1:1:1) was sufficient to catalyze the coupling with conversion and product yield of $\geq$ $95 \%$. The tnp demonstrated good catalytic properties on the product formation test platform, performing better than a traditional Pd containing mono metallic catalyst.

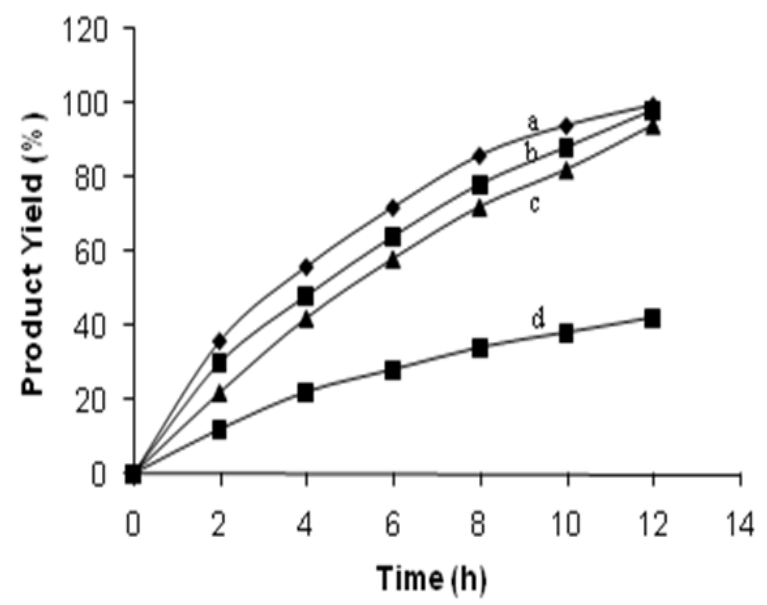

Figure 5. Comparison of the catalytic activity of $\mathrm{Au} / \mathrm{Ag} / \mathrm{Pd}$ trimetallic nanoparticles $(a=1: 1: 1, b=1: 1: 0.5)$, mono metallic Pd nanoparticles (c) and absence of nanoparticles (d)

The catalytic activity of the Pdnp and tnp towards the 
conventional Heck reaction was followed by observing the quantitative percentage yield, reaction time and turnover frequency of the product formation, methylcinnamate from the reactants bromobenzene and methyl acrylate. Optimizations of the reaction conditions such as the effects of the Pdnp catalyst and molar composition of the tnp are studied. The catalysis results are given in Fig.5. Also, tnp serves as a better catalyst for the Heck reaction, since it produced higher yields than the corresponding mono metallic palladium nanoparticle catalysts.

The turnover frequency of the catalysts has been measured by recovering the tnp precipitates are subjected Heck reaction catalysis. It is found that $99,85,46$ and 15 percent product yields existed corresponding to fresh $1^{\text {st }}, 2^{\text {nd }}$ and $3^{\text {rd }}$ reuses respectively. Catalyst reuse in the fourth time showed that, the reaction did not proceed. The metallic nanoparticles are definitely much less catalytically active during the second cycle of the Heck reaction. The reason is due to a lower amount of nanoparticles present in the solution if due to precipitation of larger nanoparticles. Another possibility is that if the number density has not changed, but only the size is getting smaller, that the smaller particles might not be as catalytically active as the larger particles. As a result, the lower catalytic activity observed during the second cycle is due to a lower amount of nanoparticles present in the solution due to larger nanoparticles aggregating and precipitating out of solution. In addition, surface poisoning by the products could be another reason.

\section{Conclusions}

In conclusion, we have synthesized a novel family of $\mathrm{Au}-\mathrm{Ag}-\mathrm{Pd}$ core-shell trimetallic nanoparticles from chemical method of metal-surfactant complexes has been reported. Surface plasmon peaks in UV-vis spectroscopy monitored the formation of mono, bi and trimetallic nanoparticles. The TEM photographs of tnp showed the sizes as $13 \pm 0.5 \mathrm{~nm}$. The particle size results from TEM agree well with each other. XPS spectra confirmed the metallic state of the colloidal nanoparticles. Their catalytic activities were tested in the Heck coupling reactions. This novel palladium containing tnp catalysis of Heck reaction produced better results than mono and bimetallic catalysts. Intact, presence of metal nanoparticles as catalysts replaces enviro-deadlier catalytic strong bases being used in Heck reaction. The high catalytic activity of trimetallic nanoparticles is probably due to the sequential electronic effect between elements in a particle. More detailed investigations of nanoparticle structure effects on the catalytic activity and their applicability in other synthetic transformations are currently under investigation.

\section{ACKNOWLEDGMENTS}

The authors thank financial support to the Department from DST-FIST.

\section{REFERENCES}

[1] Radha Narayanan, Molecules 15 (2010) 2124-2138

[2] L. Liz-Marzan, In Colloids and Colloid Assemblies; Caruso, F., Ed.; Wiley-VCH: Weinheim, 2004, p 216.

[3] P. Landon, P.J. Collier, A.J. Papworth, C.J. Kiely, G. J. Hutchings, Chem. Commun. (2002) 2058-2059

[4] J.K.Edwards, B.E. Selsone, P. Landon, A.F. Carley, A. Herzing, C.J. Kiely, G. J. Hutchings, J. Catal. 236 (2005) 69-79

[5] K. Vinodgopal, Yuanhua He, Muthupandian Ashokkumar, Franz Grieser J. Phys. Chem. B. 110, 9 (2006) 3849-3852

[6] M. Gotz, H. Wendt, Electrochim. Acta 43 (1998) 3637-3644

[7] H. Massong, H. Wang, G. Samjeske, H. Baltruschat, ibid. 46 (2000) 701-707

[8] N. Toshima, Y. Shiraishi, T. Matsushita, M. H. K. Hirakawa, Int. J Nanosci Nanotechnol. 2 (2002) 397-404.

[9] Jin Luo, Lingyan Wang, Derrick Mott, P.N Njoki, Nancy Kariuki, Chuan-Jian Zhong, T. He, J. Mater. Chem., 16 (2006) 1665-1673

[10] Viviana Benitez, Marieme Boutzeloit, V.A. Mazzieri, Catherine Especel, Florence Epron, C. R. Vera, P. Marecot, C.L. Pieck, Applied Catalysis A: General 319 (2007) 210-217

[11] Shu-Hui Tsai, Yi-Hsin Liu, Pei-Lin Wu, Chen-Sheng Yeh, J. Mater. Chem., 13 (2003) 978-980

[12] J. Santhanalakshmi, P. Venkatesan, J. Nanopar. Res. 13 (2011) 479-490

[13] P. Venkatesan and J. Santhanalakshmi, Nanoscience and Nanotechnology. 2011, 1(1), 1-5 (DOI: 10. 5923/j.nn.20110 101.01, In press)

[14] R.G.Haverkamp, A. T.Marshall, D.V.Agterveld, J. Nanopar. Res. 9 (2007) 697-700

[15] X. Zhang, F. Zhang, J. Mater. Sci. 39 (2004) 5845-5848

[16] N. Toshima, R. Ito, T. Matsushita, Y. Shiraishi, Catalysis Today 122 (2007) 239-244

[17] X. Zhang, F. Zhang, K.Y. Chan, Catal. Commun. 5 (2004) 749-753

[18] P.Venkatesan, J. Santhanalakshmi, Langmuir 26, 14 (2010) 12225-12229

[19] Toru Matsushita, Yukihide Shiraishi, Shin Horiuchi, Naoki Toshima, Bull. Chem. Soc. Jpn., 80 (2007) 1217-1225 